\title{
Eclecticismo hacia los métodịs de enseñanza-aprendizaje del español como segunda lengua en alumnos de Secundaria
}

Eclecticism towards the teaching-learning methods of Spanish as a second language in secondary school students

Ecletismo em relação aos métodos de ensino-aprendizagem do espanhol como segunda língua em alunos do ensino médio

\section{ARTÍCULO GENERAL}

\author{
Rene Altamar Manga \\ https://orcid.org/0000-0002-8609-0873 \\ renealtamar318@gmail.com
}

Universidad Internacional Iberoamericana; Arecibo Puerto Rico, EEUU

Recibido 05 de Febrero 2021 | Arbitrado y aceptado 19 de Abril 2021 | Publicado 28 de Julio 2021

\section{RESUMEN}

El objetivo de este estudio fue determinar eclécticamente cuáles son aquellos métodos y estrategias que mejorarían el proceso de enseñanzaaprendizaje de la lengua española. El estudio fue del tipo cuantitativo, cuyo diseño corresponde a estudios críticos sobre métodos de enseñanza y aprendizaje de idiomas y las estrategias específicas de cada uno de ellos. La muestra estuvo conformada por 80 estudiantes de una escuela secundaria de Massachusetts, EEUU. La técnica de recolección de datos fue el Inventario Estratégico para el Aprendizaje de Idiomas y las herramientas empleadas fueron el cuestionario y examen de Pre y Post-Prueba de nivel gramatical del español básico. Según los resultados obtenidos los mejores métodos para el aprendizaje de la lengua española se basan en que el alumno esté en mayor contacto con ella mediante actividades auditivas, visuales, conversacionales, lectivas y de escritura. Se concluye que no hay un método que se pueda denominar como el mejor. Se demostró que, a mayor exposición a la lengua extranjera, mejor es el resultado en el dominio y uso de la misma.

Palabras clave: eclecticismo; enseñanza-aprendizaje; idiomas; español; segunda lengua; alumno.

\section{ABSTRACT}

The objective of this study was to eclectically determine which are those methods and strategies that would improve the teaching-learning process of the Spanish language. The study was of the quantitative type, whose design corresponds to critical studies on language teaching and learning methods and the specific strategies of each of them. The sample consisted of 80 students from a high school in Massachusetts, USA. The data collection technique was the Strategic Inventory for Language Learning and the tools used were the questionnaire and exam of Pre and Post-Test of grammatical level of basic Spanish. According to the results obtained, the best methods for learning the Spanish language are based on the student being in greater contact with it through listening, visual, conversational, reading and writing activities. It is concluded that there is no method that can be called the best. It was shown that the greater the exposure to the foreign language, the better the result in mastering and using it.

Keywords: eclecticism; teachinglearning; Languages; Spanish; second language; pupil.

\section{RESUMO}

O objetivo deste estudo foi determinar de forma eclética quais são os métodos e estratégias que melhorariam o processo de ensino-aprendizagem da língua espanhola. $\mathrm{O}$ estudo foi do tipo quantitativo, cujo desenho corresponde a estudos críticos sobre os métodos de ensino e aprendizagem de línguas e as estratégias específicas de cada um deles. A amostra foi composta por 80 alunos de uma escola de ensino médio em Massachusetts, EUA. A técnica de coleta de dados foi o Inventário Estratégico para Aprendizagem de Línguas e as ferramentas utilizadas foram o questionário e exame de Pré e Pós-Teste de nível gramatical de espanhol básico. De acordo com os resultados obtidos, os melhores métodos para o aprendizado da língua espanhola baseiam-se no maior contato do aluno com ela por meio de atividades auditivas, visuais, conversacionais, de leitura e escrita. Conclui-se que não existe um método que possa ser denominado o melhor. Foi demonstrado que quanto maior a exposição à língua estrangeira, melhor o resultado em seu domínio e uso. Palavras-chave: ecletismo; ensinoaprendizagem; Línguas; Espanhol; segunda língua; aluno. 


\section{Introducción}

Uno de los temas en los que más se ha enfatizado en las últimas décadas en las reformas educativas de la mayoría de países, ha sido la enseñanza de una segunda lengua (Antón, 2017). No existe método ideal de enseñar o aprender una lengua (Bialystok y Hakuta, 1994), sino que es la combinación de diversas metodologías y sus posibles adaptaciones didácticas a situaciones concretas de aula lo que realmente se necesita. Es por ello que, en los últimos tiempos, el magisterio está siendo testigo de un claro eclecticismo didáctico que propone adaptarse a las necesidades concretas de cada grupo determinado de estudiantes y acepta sin complejos que lo que se está haciendo hoy en día no son más que enfoques integrados, combinaciones de metodologías pasadas que se adaptan a fin de atender las necesidades de los discentes (Dolz, Gagnon y Mosquera, 2009).

Aunque algunos autores critican que una mezcla de métodos se considere como un método en sí mismo (Martínez, 2015), las postrimerías del siglo XX han llevado a posturas como las del Postmètode (Nunan, 2004) según las cuales las diferentes metodologías han de adaptarse a las necesidades concretas de los diferentes contextos educativos y deja en manos de los docentes la decisión de escoger el procedimiento más adecuado (Monereo et al., 1999; Fernández, 2017). Sin embargo, existe un problema real: la falta de un acuerdo consensuado sobre la forma en que los alumnos utilizan las estrategias de aprendizaje de idiomas y cómo evaluar su uso. Esto atrajo la atención de varios investigadores y profesores (Bonilla y Diaz, 2018).

Para evaluar la eficacia de una determinada estrategia de aprendizaje de idiomas, la mayoría de los académicos adoptaron el Inventario Estratégico para el Aprendizaje de Idiomas (SILL) por sus siglas en inglés, de Oxford (Carson y Longhini, 2002; Nisbet, Tindall y Arroyo, 2005). Mientras tanto, al darse cuenta de ciertas limitaciones de SILL, algunos académicos reflexionaron sobre nuevos enfoques para evaluar el uso de estrategias de aprendizaje de idiomas (Tseng, Dörnyei y Schmitt, 2006). Woodrow (2016) discutió la utilidad de las escalas de tipo Likert para evaluar las estrategias de aprendizaje de idiomas, descubriendo que no era apropiado y propuso un nuevo enfoque utilizando métodos cualitativos.

Los diferentes estudiantes tienen la posibilidad de utilizar diversos métodos con o sin éxito, dado que hay factores de variedad detrás de ello que influyen en el uso de la 
estrategia de aprendizaje de idiomas. Estos factores afectan al número, frecuencia y tipos de estrategias de aprendizaje de idiomas, entre ellos se encuentran: antecedentes culturales, idioma estudiado, etapa de aprendizaje, edad, motivación, objetivos de aprendizaje de idiomas, entornos de la lengua materna frente a segunda lengua, experiencia previa de aprendizaje de idiomas, estilos de aprendizaje de idiomas, género, ansiedad, falta de inhibición y especialización profesional o académica (Manga, 2008; 2016; Santana, García y Escalera, 2016).

Dado que el uso de estrategias de aprendizaje de idiomas ha demostrado ser útil y beneficioso en la mayoría de los casos, las estrategias de aprendizaje de idiomas de los alumnos exitosos podrían enseñarse o compartirse con los menos exitosos, en otras palabras, cabe preguntarnos si tales estrategias de idiomas son enseñables. En fusión a esto, una minoría de estudiosos tiene puntos de vista negativos (Rees, 1993; Macaro, 2006; Plonsky, 2011; Chamot y Harris, 2019). "Los estudios sobre las IS (Instructional Strategies) también han producido resultados negativos y mixtos en muchos de los mismos contextos, tratamientos y variables de resultado" (Plonsky, 2011: p. 994).

En cualquier caso, es aconsejable que los profesores comprendan a fondo las estrategias de aprendizaje de idiomas para poder enseñar a los estudiantes a adoptar los métodos adecuados impulsando así a su aprendizaje (Qin y Buripakdi, 2016; Thornbury, 2017).

En relación a esa falta de consenso sobre las estrategias de enseñanza-aprendizaje de inglés como segundo idioma, este estudio busca responder a las siguientes preguntas:

1. ¿Cómo es la actitud del alumno, su práctica de la segunda lengua, su preparación, su aptitud, el contacto con la segunda lengua, su disposición hacia actividades de estudio de la segunda lengua en el aula?

2. ¿Cuáles deben ser los métodos, actividades y estrategias a utilizar para la enseñanza de la segunda lengua en el salón de clases?

El objetivo de esta investigación es estudiar eclécticamente cuáles son aquellos métodos y estrategias que mejorarían el proceso de enseñanza-aprendizaje de la lengua española y constatar su resultado en la Escuela Secundaria en Estados Unidos. 


\section{Estado del arte}

La mayoría de los habitantes del planeta son bilingües y muchos aprendieron su segunda lengua cuando ya eran mayores, lo que indica que la falta de habilidad se puede suplir con trabajo y constancia. El alumno para tener éxito, debe fijar motivación; es importante recalcar la necesidad de realizar un esfuerzo, y no presentarlo como una tarea aburrida sino como una inversión (López, 2006). Si a una persona le gusta el contenido del tema, se sentirá inmediatamente motivada y tendrá ganas de aprender tanto como pueda sobre dicho tema (Sánchez y Vargas, 2016; González y Rodríguez, 2017).

Hasta el momento no se conoce un método perfecto de aprender un idioma, el dominio de una segunda lengua puede conseguirse de varias maneras utilizando diversas estrategias. El descubrimiento de las mejores técnicas para el aprendizaje por parte de los estudiantes es el factor primordial para el éxito (Plasencia, 2020).

Dansereau (1979) ya resaltaba que las estrategias de aprendizaje están vinculadas estrechamente a la consecución de un aprendizaje efectivo, en conjunto con una instrucción de calidad y estrategias que motiven al estudiante, teniendo en cuenta tanto la metodología didáctica (Tiberius, 2010) como hacer que se sienta motivado para aprender más sobre el tema por sí mismo (Sampedro, 2019).

Guerrero (2013) señala que un proyecto de enseñanza de segunda lengua debe partir de un análisis previo del contexto general en el que se incluye (social, educativo, cultural, emocional) que permita responder a las exigencias de las etapas de desarrollo social de los alumnos y a las necesidades de utilización de la segunda lengua.

El modelo integrador de Stern et al. (1983) muestra una serie de variables entre las que destacan las relativas al contexto social (variables sociolingüísticas), a las características del aprendiz (variables cognitivas y afectivas), a las condiciones del aprendizaje (variables relacionadas con el sistema educativo y/o con la exposición a la segunda lengua), al proceso de aprendizaje (operaciones mentales y estrategias) y al resultado del aprendizaje (competencia alcanzada). Al Shehri (2012) señala que este modelo facilita y consolida el uso de las estrategias comunicativas.

Según Chastain (1970), tanto los estudiantes como los profesores y los componentes del lenguaje en sí mismo, son tan diversos que no justifican la utilización 
de un solo método. Lo mejor sería preguntarse qué enfoque debe aplicarse, con cuáles profesores, con cuáles estudiantes y para cuáles aspectos del lenguaje.

No existe una metodología pura para la enseñanza de una segunda lengua, y por ello es lícito un eclecticismo en cuanto a ella y cada profesor sabrá cuál resulta eficaz para su grupo clase (Valenzuela, Romero, Vidal y Philominraj, 2016).

En su estudio, Singer, Rubio y Rubio (2019) señalan que los alumnos de niveles avanzados son capaces de desarrollar estrategias de aprendizaje y adoptar metodologías más activas. Para Macías, Torres, Zambrano y Asang (2016) las tecnologías toman el control de muchas actividades de la vida cotidiana, y su implementación para apoyar el trabajo en clase constituye un aspecto novedoso para los alumnos; siendo en ocasiones necesario desprenderse de la enseñanza tradicionalista, sobre todo en el ámbito de idiomas. Utilizando la música como herramienta estratégica, Cores (2019) señala que los alumnos maximizan sus habilidades socio-emocionales y comunicativas. Ellison, Pazos, Martínez y Rodrigues (2018) recomiendan la incorporación de una didáctica lexicográfica en el aula de lengua extranjera para suplir las necesidades concretas de los usuarios debiendo incluir los nuevos formatos virtuales.

En congruencia con lo anterior, se diseñó un estudio cuantitativo sobre los métodos y estrategias que se han utilizado tradicionalmente en las aulas de lenguas extranjeras, con la finalidad de constatar cuales son los métodos de mayor efectividad según los alumnos.

\section{Metodología}

\subsection{Tipo y diseño de estudio}

Se realizó un estudio cuantitativo, cuyo diseño corresponde a estudios críticos sobre métodos y estrategias para enseñar la segunda lengua, el diseño metodológico corresponde a encuestas preparadas con base en encuestas utilizadas en investigaciones sobre la segunda lengua, teniendo como principal referencia la denominada SILL. Se hizo una serie de adaptaciones de forma que las preguntas fuesen más manejables para la muestra de estudio. El análisis de datos se realizó mediante Microsoft Excel y SPSS 25.0 
Se realizaron dos encuestas:

La primera tenía como objetivo responder a la primera pregunta de investigación: ¿Cómo es la actitud del alumno, su disponibilidad, su preparación, su aptitud, el contacto con la segunda lengua, su disposición hacia actividades de estudio de la segunda lengua en el aula?

La segunda encuesta se concentra en dos áreas: los métodos, actividades y estrategias que se han empleado siempre en el salón de clases para impartir la enseñanza de la segunda lengua. En esta aparecen nombres de los métodos más renombrados para la enseñanza de la segunda lengua; asimismo, se encuentra enumerado un número significativo de actividades de enseñanza y aprendizaje de la segunda lengua que siempre se han utilizado en el aula de clases de lenguas extranjeras.

La hipótesis del estudio fue: el estudiantado de la escuela secundaria mejorará en su estudio de la segunda lengua, con una aplicación ecléctica de los mejores métodos y estrategias en la enseñanza de la segunda lengua.

\section{2. Instrumento de recolección de datos}

Para la recopilación de datos se aplicó cuestionarios a los estudiantes; la encuesta SILL; examen de Pre-Prueba de nivel gramatical del español básico; rúbricas de tareas y actividades; ejercicios estructurados; tareas de completar; examen de aprovechamiento o Post-Prueba.

\section{3. Actividades realizadas}

Se llevó a cabo traducción de palabras y oraciones; comprensión lectora; comprensión auditiva; actividad comunicativa; escritura de oraciones y párrafos.

\section{4. Muestra del estudio}

Se realizó un muestreo no probabilístico (por conveniencia). La muestra estuvo conformada por 80 estudiantes de una escuela secundaria del pueblo de Southbridge, Massachusetts, EEUU. Los años o niveles académicos de los estudiantes participantes en el estudio investigativo fluctúan entre el noveno grado y el décimo-segundo grado de la escuela secundaria. El estudiantado participante en el estudio habla inglés como lengua materna. 


\section{5. Criterios de inclusión}

Rene Altamar Manga

- Ser angloparlantes

- No haber tenido preparación previa en español

- Estar cursando el primer año de español

- Tener entre 14 y 18 años de edad

- Haber nacido en el estado de Massachusetts o algún otro estado vecino de la región de New England, en el noreste de Estados Unidos.

\subsection{Criterios de exclusión}

- Ser de origen hispano

- Ser estudiante clasificado como alumno de educación especial.

\section{Resultados}

En cuanto a la hipótesis de estudio, luego de la aplicación ecléctica de los mejores métodos y estrategias en la enseñanza de la segunda lengua, como se puede apreciar en la tabla mostrada a continuación, la menor calificación para la preprueba la obtuvo el décimo grado, mientras que la mayor la obtuvo el décimo segundo, del mismo modo para la posprueba la mayor calificación la obtuvo el décimo segundo grado, pero en este caso la menor fue para noveno grado.

Tabla 1. Resumen de los puntajes obtenidos en la preprueba y posprueba

\begin{tabular}{llll}
\hline & Grado & Preprueba & Posprueba \\
\hline Promedio & 9 & 70.4 & 74.9 \\
& 10 & 69.5 & 77.0 \\
& 11 & 72.5 & 77.4 \\
Desviación estándar & 9 & 82.3 & 87.6 \\
& 12 & 11.9 & 8.61 \\
& 11 & 11.2 & 10.7 \\
Mínimo & 12 & 5.77 & 5.16 \\
& 9 & 50.0 & 53.0 \\
& 10 & 45.0 & 55.0
\end{tabular}




\begin{tabular}{llll} 
& 11 & 47.0 & 51.0 \\
Máximo & 12 & 75.0 & 80.0 \\
& 9 & 83.0 & 90.0 \\
& 10 & 88.0 & 93.0 \\
& 11 & 90.0 & 100 \\
12 & 90.0 & 96.0 \\
\hline
\end{tabular}

\section{1. Prueba de hipótesis}

Se compararon los puntajes pre y posprueba con la prueba T de Student, con un nivel de significancia del 5\% y se trabajó con valores a dos colas. Se obtuvieron diferencias estadísticamente significativas en los puntajes en la preprueba y la postprueba para todos los grados. En vista de lo anterior, se rechaza la hipótesis nula que enuncia, que el puntaje de la posprueba es igual que el puntaje de la preprueba. Se acepta la hipótesis alterna, que enuncia, que el puntaje de la posprueba es diferente al puntaje de la preprueba. Los estudiantes mejoraron sus estudios de la segunda lengua con una mejor aplicación ecléctica de los métodos y estrategias que sean de su preferencia. Al lograr aplicar eclécticamente lo mejor de los métodos y estrategias de enseñanza, tomando en cuenta cómo se aprende la primera lengua, la segunda lengua y la preferencia metodológica y estratégica para el proceso de enseñanza-aprendizaje, se puede asegurar con certeza que el alumno tendrá mejor éxito en la adquisición de la segunda lengua.

Para responder a las preguntas de investigación se presentan a continuación, una serie de tablas que resumen las principales respuestas:

Tabla 2. Motivación de los estudiantes para aprender la segunda lengua

\begin{tabular}{ccc}
\hline $\begin{array}{c}\text { Nivel de } \\
\text { motivación }\end{array}$ & $\mathrm{n}$ & $\%$ \\
\hline Bajo & 12 & 14.12 \\
Medio & 19 & 22.35 \\
Alto & 48 & 56.47 \\
Sin contestar & 6 & 7.06 \\
Total & 85 & 100 \\
\hline
\end{tabular}


Tabla 3. Actitud del estudiante para aprender la segunda lengua

\begin{tabular}{ccc}
\hline $\begin{array}{c}\text { Estudiante tiene actitud positiva e iniciativa propia para aprender } \\
\text { la segunda lengua }\end{array}$ & $\mathrm{n}$ & $\%$ \\
\hline Si & 68 & 80. \\
& & 16. \\
No & 14 & 47 \\
& & 3.5 \\
Sin contestar & 3 & 3 \\
Total & 85 & 100 \\
\hline
\end{tabular}

El $80 \%$ de los alumnos indicaron tener una actitud positiva e iniciativa propia para aprender la segunda lengua.

Tabla 4. Preparación obtenida de la segunda lengua

\begin{tabular}{lcc}
\hline Preparación & $\mathrm{n}$ & $\%$ \\
\hline \multicolumn{1}{l}{ Estudiante ha tomado un examen de nivel clasificatorio en la segunda lengua } \\
\hline $\mathrm{Si}$ & 13 & 15.30 \\
$\mathrm{No}$ & 70 & 82.35 \\
Sin contestar & 2 & 2.35 \\
Total & 85 & 100 \\
\hline Estudio de la segunda lengua con amigo o tutor & \\
\hline $\mathrm{Si}$ & 30 & 35.29 \\
$\mathrm{No}$ & 52 & 61.18 \\
Sin contestar & 3 & 3.53 \\
Total & 85 & 100 \\
\hline Estudio de la segunda lengua mediante el internet & \\
\hline Si & 35 & 41.18 \\
No & 48 & 56.47 \\
Sin contestar & 2 & 2.35 \\
Total & 85 & 100 \\
\hline
\end{tabular}

Tabla 5. Disposición del estudiante para utilizar su nivel de inglés para comunicarse

\begin{tabular}{lll}
\hline $\begin{array}{l}\text { El estudiante está dispuesto a usar lo que sabe en la segunda } \\
\text { lengua para comunicarse }\end{array}$ & $\mathrm{n}$ & $\%$ \\
\hline & & 71. \\
$\mathrm{Si}$ & 61 & 77 \\
& & 25. \\
No & 22 & 88 \\
& & 2.3 \\
Sin contestar & 2 & 5 \\
Total & 85 & 100 \\
\hline
\end{tabular}


Tabla 6. Contacto con la segunda lengua

\begin{tabular}{lll}
\hline $\begin{array}{l}\text { Estudiante trata de entender la segunda lengua cuando es hablada } \\
\text { por hablantes nativos }\end{array}$ & $\mathrm{n}$ & $\%$ \\
\hline & & 52. \\
$\mathrm{Si}$ & 45 & 94 \\
& & 42. \\
$\mathrm{No}$ & 36 & 35 \\
& & 4.7 \\
Sin contestar & 4 & 1 \\
Total & 85 & 100 \\
\hline
\end{tabular}

El 52,94\% de los alumnos indicaron tratar de entender la segunda lengua cuando era hablada por hablantes nativos. Aunado a esto el 11,76 \% de los alumnos indicaron haber viajado a algún país donde se hablaba la segunda lengua que estudiaba lo que les permitió en ese caso aprender más.

Tabla 7. Frecuencia de participación en clase

\begin{tabular}{lllll}
\hline $\begin{array}{l}\text { Actividades realizadas } \\
\text { en las Clases }\end{array}$ & A veces & Siempre & Nunca & Sin contestar \\
\hline Actividades del Texto & 40 & 32 & 10 & 3 \\
Diálogos & 37 & 40 & 5 & 3 \\
Reglas Gramaticales & 31 & 38 & 9 & 7 \\
Trabajo en grupo & 29 & 39 & 9 & 8 \\
Memorización & 33 & 44 & 5 & 3 \\
Repetición & 28 & 44 & 8 & 5 \\
Audio & 25 & 50 & 6 & 4 \\
Video & 34 & 45 & 5 & 1 \\
Escritura & 19 & 53 & 7 & 6 \\
Lectura & 30 & 44 & 9 & 2 \\
Micrófono & 27 & 44 & 8 & 6 \\
\hline
\end{tabular}

Las actividades en clase en las que más participaban los alumnos a veces o siempre eran los diálogos, la memorización, el audio y el video. 
Tabla 8. Mejor método para aprender una segunda lengua en alumnos

\begin{tabular}{|c|c|c|c|c|c|}
\hline $\begin{array}{l}\text { Método para aprender } \\
\text { una segunda lengua }\end{array}$ & $\begin{array}{l}\text { El } \\
\text { mejor }\end{array}$ & $\begin{array}{l}\text { Muy } \\
\text { bueno }\end{array}$ & Good & $\begin{array}{l}\text { Buen } \\
\text { promedio }\end{array}$ & El peor \\
\hline \multicolumn{6}{|c|}{ Alumnos de noveno grado } \\
\hline Grammar Translation & 8 & 6 & 5 & 6 & 1 \\
\hline Direct Method & 7 & 11 & 7 & 1 & 0 \\
\hline Audiolingual & 11 & 8 & 7 & 1 & 0 \\
\hline Immersion & 4 & 12 & 6 & 5 & 0 \\
\hline Total Physical Response & 1 & 6 & 9 & 6 & 4 \\
\hline Natural Approach & 6 & 15 & 3 & 2 & 0 \\
\hline Computer Assisted & 10 & 7 & 9 & 0 & 0 \\
\hline Communicative & 4 & 15 & 6 & 1 & 0 \\
\hline Task Based & 6 & 9 & 8 & 3 & 0 \\
\hline ContentBased & 3 & 7 & 13 & 3 & 0 \\
\hline \multicolumn{6}{|l|}{ Alumnos de décimo grado } \\
\hline Grammar Translation & 9 & 5 & 5 & 4 & 2 \\
\hline Direct Method & 6 & 9 & 5 & 3 & 3 \\
\hline Audiolingual & 6 & 5 & 7 & 4 & 3 \\
\hline Immersion & 5 & 9 & 6 & 4 & 3 \\
\hline Total Physical Response & 4 & 7 & 7 & 5 & 3 \\
\hline Natural Approach & 6 & 7 & 7 & 4 & 2 \\
\hline Computer Assisted & 6 & 9 & 7 & 3 & 2 \\
\hline Communicative & 6 & 8 & 8 & 4 & 1 \\
\hline Task Based & 6 & 8 & 5 & 3 & 3 \\
\hline ContentBased & 4 & 8 & 7 & 4 & 3 \\
\hline \multicolumn{6}{|c|}{ Alumnos de décimo primer grado } \\
\hline Grammar Translation & 6 & 5 & 0 & 1 & 0 \\
\hline Direct Method & 5 & 5 & 2 & 0 & 0 \\
\hline Audiolingual & 3 & 4 & 3 & 1 & 0 \\
\hline Immersion & 4 & 2 & 1 & 1 & 2 \\
\hline Total Physical Response & 1 & 3 & 2 & 5 & 2 \\
\hline Natural Approach & 5 & 3 & 2 & 1 & 0 \\
\hline Computer Assisted & 3 & 6 & 1 & 2 & 1 \\
\hline Communicative & 2 & 3 & 5 & 1 & 0 \\
\hline Task Based & 3 & 4 & 2 & 1 & 0 \\
\hline ContentBased & 2 & 2 & 5 & 1 & 1 \\
\hline \multicolumn{6}{|c|}{ Alumnos de décimo segundo grado } \\
\hline Grammar Translation & 5 & 4 & 1 & 0 & 0 \\
\hline Direct Method & 3 & 6 & 1 & 0 & 0 \\
\hline Audiolingual & 2 & 4 & 4 & 1 & 0 \\
\hline Immersion & 1 & 2 & 4 & 3 & 0 \\
\hline Total Physical Response & 1 & 3 & 2 & 4 & 1 \\
\hline Natural Approach & 2 & 3 & 3 & 1 & 1 \\
\hline Computer Assisted & 3 & 4 & 2 & 1 & 0 \\
\hline Communicative & 2 & 4 & 2 & 2 & 0 \\
\hline Task Based & 2 & 4 & 4 & 0 & 0 \\
\hline ContentBased & 3 & 4 & 1 & 1 & 1 \\
\hline
\end{tabular}


Los alumnos de noveno grado consideraron como mejor método el audiolingüe y el computer assisted, principalmente. Las mejores estrategias para aprender una segunda lengua fueron recognition of rewards, games, less focus on grammar, lang practice, flash cards, posters, bingos y entertainment.

Los alumnos de décimo grado consideraron como mejor método al grammar translation sobre todos los otros métodos. Las mejores estrategias para aprender una segunda lengua fueron flashcards, use of basic expressions, vocab review, walkietalkies, posters y entertainment.

Los alumnos de décimo primer grado consideraron como mejor método al gramar translation principalmente, seguido de natural approach y direct method por igual. Consideraron como mejor estrategia para aprender una segunda lengua games, entertainment, bingos, posters, rest menus and recipes.

Los alumnos de décimo segundo grado consideraron como mejor método al grammar translation y direct method. Y consideraron como mejor estrategia para aprender una segunda lengua flashcards, bingos, entertainment, vocab review, repetition y translation.

\section{Discusión}

Es necesario discutir algunos aspectos de gran relevancia respecto a la enseñanza y aprendizaje de la segunda lengua, con una aplicación ecléctica de los mejores métodos y estrategias para su enseñanza. En esta investigación se presentaron dos interrogantes a las que se buscó responder. La primera referida a la actitud del alumno, su disponibilidad, preparación, aptitud, el contacto con la segunda lengua, su disposición a participar en actividades de estudio de la segunda lengua en el aula. Un 56.47 \% tenía una motivación alta, seguido de un 22,35\% con motivación media. Para Oga, Nakata, Parker y Ryan (2017) existe una relación dinámica y positiva entre las percepciones de aprendizaje y la motivación, afirmando que el compromiso de los alumnos fue el augurador situacional de una actitud motivada para el aprendizaje de la segunda lengua. De acuerdo con Lamb (2017) la motivación es identificada como un factor vital en el éxito del aprendizaje de una segunda lengua y ha sido centro de múltiples investigaciones en los últimos tiempos, asegurando a su vez que con demasiada 
frecuencia es la desmotivación del estudiante la causa del resultado no deseado en la enseñanza de un segundo idioma.

La mayoría de alumnos tenía una actitud positiva e iniciativa propia para aprender la segunda lengua y estaban dispuestos a usar lo que sabían de la segunda lengua para comunicarse. Para dichos efectos es relevante resaltar a Sánchez y Vargas (2016) y González y Rodríguez (2017) quienes afirman que, si a una persona le gusta el contenido del tema, se sentirá inmediatamente motivada y tendrá ganas de aprender tanto como pueda sobre dicho tema. Del mismo modo Arellano, Reinao, Marianjel y Curaqueo (2020) aseguran que factores a los que se debe, de forma parcial el éxito del proceso de aprendizaje de una segunda lengua son la motivación, la aptitud y la personalidad. También es importante destacar que el puntaje promedio en la posprueba fue mayor, con una diferencia estadísticamente significativa que el de la preprueba en todos los grados. Los estudiantes mejoraron sus estudios de la segunda lengua con una mejor aplicación ecléctica de los métodos y estrategias que fueron de su preferencia, los cuales se mencionan a continuación: Grammar Translation y Direct Method como métodos de preferencia; Flash Cards, Bingos, Entertainment y Posters como estrategias de preferencia. En este sentido Según Alharbi (2017) es de marcada necesidad la utilización de múltiples enfoques de enseñanza cuando se trata de idiomas, orientada a una verdadera conexión con todos los estudiantes para así poder cubrir las necesidades individuales a través de distintas pedagogías pudiendo abordar marcadas dificultades en el estudiantado y examinar y evaluar de manera crítica las formas convencionales.

La segunda interrogante se refirió a los métodos, actividades y estrategias que se han utilizado en la enseñanza de la segunda lengua, enlistando un número significativo de actividades de enseñanza y aprendizaje que siempre se han utilizado en el aula de clases de lenguas extranjeras. Un poco más de la mitad de los alumnos $(52,94 \%)$ trataban de entender la segunda lengua cuando era hablada por hablantes nativos. Según Arellano, et al. (2020) estas iniciativas donde son involucrados hablantes nativos de la lengua estudiada que participan del proceso educativo resulta un método de alta aceptación por expertos lingüistas quienes aseguran que es una de las estrategias más eficaces y apropiadas para el aprendizaje de nuevos idiomas, capaz de revitalizarlo de manera significativa. Esto lo ejemplifica Branda (2017) en su investigación asegurando que nativos de habla hispana residenciados en países de habla inglesa, si bien suelen no 
utilizar inglés cuando interactúan en su entorno familiar, lo usan interactuando en su vida laboral y social dentro del país que habitan, nutriéndolo cada vez más.

Por otro lado, pocos alumnos (15,29\%) manifestaron haber viajado a algún país donde se hablaba la segunda lengua que estudiaba y aprendía, y sólo un 11,76\% indicó haber tomado un examen de nivel clasificatorio en la segunda lengua. En cuanto a la participación en las actividades realizadas en clase, los alumnos participaron a veces o siempre en los diálogos, la memorización, el audio y el video. En tal sentido Ellison, et al. (2018) recomiendan la incorporación de una didáctica lexicográfica en el aula de lengua extranjera para suplir las necesidades concretas de los usuarios debiendo incluir los nuevos formatos virtuales. Según Ignatova, Kalyuga y Sweller (2020) afirman que la carga cognitiva producto de la imaginación conjuntamente con la utilización de recursos visuales y espaciales mejora el aprendizaje de una segunda lengua. En tal sentido Bilyalova (2017) también sugiere que una de las formas de mayor efectividad orientada a mejorar la calidad de la enseñanza de la segunda lengua es la informatización como método de enseñanza, la cual personaliza e intensifica el aprendizaje evitando la valoración subjetiva, por lo tanto, las herramientas digitales pueden emplearse de manera eficaz en las lecciones de nuevos idiomas.

En función a todo lo anteriormente mencionado se puede decir que no hay metodología que contenga sólo un método para la enseñanza de una segunda lengua, coincidiendo en este sentido con Valenzuela, et al. (2016) quienes afirman que no existe una metodología pura para la enseñanza de una segunda lengua, por ello es lícito un eclecticismo en cuanto a ella y cada profesor sabrá cuál resulta eficaz para su grupo clase. Benito (2019) afirma en su estudio que se ha pasado de hablar de métodos de enseñanza-aprendizaje de lenguas a enfoques, y en estos últimos uno de los adjetivos más utilizados es ecléctico, enfoque ecléctico, constatando la necesidad de elaborar propuestas prácticas. Es por ello que Alharbi (2017) apoya y promueve la utilización del enfoque ecléctico por parte de los profesores en la enseñanza, adaptándola a la metodología tradicional para que el aprendizaje se vuelva productivo, práctico y efectivo, también reconocido entre otros expertos en enseñanza de idiomas como ecléctico de principios. No obstante, Jabbarova (2020) indica que a pesar de que el método ecléctico es un método popular en la actualidad debido a la heterogeneidad y versatilidad propias del estudiante moderno, existe especial sensibilidad en algunos profesores en la utilización de una multiplicidad de métodos, a quienes les resulta de 
mayor comodidad el uso de un método en particular dentro del aula. Por lo que se establece al profesor como el factor indispensable en la selección de la metodología para la enseñanza de una segunda lengua (Arellano et al., 2020).

\section{Conclusión}

Se concluye que no hay un método que se pueda denominar como el mejor, puesto que cada método con sus respectivas estrategias aporta en mayor o menor grado un elemento positivo hacia el éxito de la enseñanza y el aprendizaje total de una segunda lengua, no obstante, se demostró que, a mayor exposición en la lengua extranjera, mejor el resultado en el dominio y uso de la misma. Los alumnos manifestaron preferencia sobre los métodos y estrategias que se mencionan a continuación: Grammar Translation y Direct Method como métodos de preferencia; Flash Cards, Bingos, Entertainment y Posters como estrategias de preferencia.

Se pudo constatar que, aplicar eclécticamente lo mejor de los métodos y estrategias de enseñanza, tomando en cuenta cómo se aprende la primera lengua, la segunda lengua y la preferencia metodológica y estratégica para el proceso de enseñanza-aprendizaje, se puede asegurar con certeza que el alumno tendrá mejor éxito en la adquisición de la segunda lengua.

Esta investigación sienta las bases para desarrollar programas académicos de enseñanza y aprendizaje de las lenguas clásicas griego y latín, así como también de otras lenguas contemporáneas como el alemán, el español, el francés, el italiano, el portugués y el rumano.

\section{Referencias bibliográficas}

Alharbi, S. H. 2017. Principled Eclecticism: Approach and Application in Teaching Writing to ESL/EFL Students. English Language Teaching, 10(2), 33-39.

Al Shehri, H. 2012. Los modelos de adquisición y enseñanza en una segunda lengua, y la hipótesis del filtro afectivo de Krashen. Avances en Supervisión Educativa, (16).

Antón, M. 2017. Segunda lengua, evaluación dinámica e inclusión educativa en Estados Unidos. Revista de Educación Inclusiva, 3(1). 
Arellano, R., P. Reinao, A. Marianjel Y G. Curaqueo. 2020. Un estudio comparativo entre las metodologías usadas en la enseñanza del mapudungun como segunda lengua y el inglés como lengua extranjera. Forma y Función, 33(1), 87-114. DOI: https://doi.org/10.15446/fyf.v33n1.79657

Benito, A. 2019. Vuelta a escena de la traducción en la enseñanza de lenguas extranjeras: experiencias en PLE. Hermeneus: Revista de la Facultad de Traducción e Interpretación de Soria, (21), 197-234.

Bialystok, E., Y K. Hakuta. 1994. In other words. New York: Basic Books.

Bilyalova, A. 2017. ICT in teaching a foreign language in high school. ProcediaSocial and Behavioral Sciences, 237, 175-181.

Bonilla, M. Y C. Díaz. 2018. La metacognición en el aprendizaje de una segunda lengua: Estrategias, instrumentos y evaluación. Revista Educación, 42(2), 629644. https://dx.doi.org/10.15517/revedu.v42i2.25909

Branda, S. 2017. Los distintos enfoques en la enseñanza de inglés como lengua extranjera y segunda: nuevas tendencias en la literatura contemporánea. Revista de Educación, 2(11), 99-112.

Carson, J., Y A. Longhini. 2002. Focusing on learning styles and strategies: A diary study in an immersion setting. Language learning, 52(2), 401-438.

Chamot, A. Y V. Harris. (Eds.). 2019. Instrucción de estrategia de aprendizaje en el aula de idiomas: problemas e implementación . Asuntos multilingües.

Chastain, K. 1970. Behavioristic and cognitive approaches in programmed instruction. Language learning, 20(2), 223-235. https://doi.org/10.1111/j.1467-

\subsection{0.tb00479.x}

Cores-Bilbao, E., A. Fernández-Corbacho., F. Machancoses Y M. Fonseca-Mora. 2019. A music-mediated language learning experience: Students' awareness of their socio-emotional skills. Frontiers in psychology, 10, 2238.

https://doi.org/10.3389/fpsyg.2019.02238 
Dansereau, D., K. Collins, B. Mcdonald, C. Holly, J. Garland, G. Diekhoff, Y S. Evans. 1979. Development and evaluation of a learning strategy training program. Journal of Educational Psychology, 71(1), 64. 383 citas.

Dolz, J., R. Gagnon Y S. Mosquera. 2009. La didáctica de las lenguas: una disciplina en proceso de construcción. Didáctica. Lengua y literatura, 21, 117-141.

Ellison, M., M. Pazos, P. Nicolás Y S. Rodrigues. 2018. As línguas estrangeiras no ensino superior: propostas didáticas e casos em estudo.

Fernández, R. 2017. Dimensiones del trabajo cotidiano en tensión para la autonomía docente Un estudio de carácter etnográfico sobre las dimensiones en tensión de la autonomía docente y la toma de decisiones en el trabajo cotidiano en distintos contextos educativos.

González, M., Y Rodríguez, B. 2017. Factores motivacionales de los adultos para el estudio de una lengua extranjera. Pedagogía Social. Revista Interuniversitaria, (30), $129-143$

Guerrero, R. 2013. Integración sociocultural y enseñanza del español en Andalucía: Aplicación del programa Aulas Temporales de Adaptación Lingüística (ATAL). Perfiles educativos, 35(142), 42-53.

Ignatova, O., S. Kalyuga Y J. Sweller. 2020. The imagination effect when using textual or diagrammatic material to learn a second language. Language Teaching Research. https://doi.org/10.1177/1362168820971785

Jabbarova, A. 2020. The importance of the teaching method-theory and its application. Архив Научных Публикаций JSPI.

Lamb, M. 2017. The motivational dimension of language teaching. Language Teaching, 50(3), 301-346.

López, J. 2006. Aportaciones de la teoría de las atribuciones causales a la comprensión de la motivación para el rendimiento escolar. Ensayos: Revista de la Facultad de Educación de Albacete, (21), 217-232. 
Macaro, E. 2006. Strategies for language learning and for language use: Revising the theoretical framework. Modern language journal, 90(3), 320-337.

https://doi.org/10.1111/j.1540-4781.2006.00425.x

Macías, R., J. Torres, J. Zambrano Y A. Asang. 2016. Juegos virtuales interactivos para desarrollar las habilidades lingüísticas en estudiantes de bachillerato en la unidad educativa "Eloy Alfaro" de Bahía de Caraquez.Revista Didasc@lia:

Didáctica y Educación, 7(2).

Manga, A. 2008. Lengua Segunda (L2) Lengua extranjera (LE): Factores e incidencias de enseñanza/aprendizaje. Tonos digital, 16(0).

Martínez, J. 2015. La selección del método en la investigación jurídica. 100 métodos posibles. Revista de Educación y Derecho., (12).

https://doi.org/10.1344/re\&d.v0i12.14402

Monereo, C., M. Castelló, M. Clariana, M. Palma, Y M. Pérez. 1999. Estrategias de enseñanza y aprendizaje. Formación del profesorado y aplicación en la escuela. $6^{\text {ta }}$ Ed. Editorial Graó. Barcelona.

Nisbet, D., E. Tindall Y A. Arroyo. 2005. Language learning strategies and English proficiency of Chinese university students. Foreign language annals, 38(1), 100-107.

Nunan, D. 2004. Task-based language teaching. Ernst Klett Sprachen. https://bestofbilash.ualberta.ca/Task-based\%20Language \%20Teaching.pdf

Oga-Baldwin, W , Y. Nakata, P. Parker, Y R. Ryan. 2017. Motivating young language learners: A longitudinal model of self-determined motivation in elementary school foreign language classes. Contemporary Educational Psychology, 49, 140-150.

Plasencia, Z. 2020. Elementos que intervienen en el éxito-y en el fracaso-de la interacción oral en lengua extranjera en secundaria. DIGILEC: revista internacional de lenguas y culturas, 6, 14-26.

Plonsky, L. 2011. The effectiveness of second language strategy instruction: A meta-analysis. Language learning, 61(4), 993-1038. https://doi.org/10.1111/j.14679922.2011.00663.x 
Qin, X. Y A. Buripakdi. Junio de 2016. Un análisis del discurso de los libros de texto de inglés de la escuela secundaria china: componentes culturales y construcción ideológica. En la 4ta Conferencia FLLT .

Rees-Miller, J. 1993. A critical appraisal of learner training: Theoretical bases and teaching implications. TESOL quarterly, 27(4), 679-689. 317.

Sampedro, M. 2019. Motivenglish 1: La motivación en el aula de inglés de primaria y secundaria. Campo Abierto. Revista de Educación, 38(1), 35-44.

Sánchez, M. Y M. Vargas. 2016. El alumno motivado: un análisis empírico de los factores motivadores intrínsecos y extrínsecos en el aula de inglés. Investigación en la Escuela, (90).

Santana Villegas, J., A. García-Santillán, Y M. Escalera-Chávez. 2016. Variables que influyen sobre el aprendizaje del inglés como segunda lengua. Revista Internacional de Lenguas Extranjeras / International Journal of Foreign Languages, 0(5), 79-94. doi:https://doi.org/10.17345/rile5.1004

Singer, N., M. Rubio, Y R. Rubio. 2019. Ensino de línguas: uma comparação das representações sociais de alunos do primeiro e quinto anos da carreira de Lingüística Aplicada à Tradução. DELTA: Documentação de Estudos em Lingüística Teórica e Aplicada, 35(4). https://dx.doi.org/10.1590/1678-460x2019350407

Stern, H., E. Tarone, H. Stern, G. Yule, Y H. Stern. 1983. Conceptos fundamentales de la enseñanza de idiomas: perspectivas históricas e interdisciplinarias sobre la investigación lingüística aplicada. Prensa de la Universidad de Oxford.

Thornbury, S. 2017. Los 30 métodos de enseñanza de idiomas de Scott Thornbury Google EBook: manuales de Cambridge para profesores de idiomas. Prensa de la Universidad de Cambridge.

Tiberius, V. 2010. Didactics of future studies. Journal of Future Studies, 14(4), 135-146.

Tseng, W, Z. Dörnyei, Y N. Schmitt. 2006. A new approach to assessing strategic learning: The case of self-regulation in vocabulary acquisition. Applied linguistics, 27(1), 78-102. 
Valenzuela, M., K. Romero, C. Vidal-Silva, Y A. Philominraj. 2016. Factores que Influyen en el aprendizaje del idioma inglés de nivel inicial en una universidad chilena. Formación universitaria, 9(6), 63-72. http://dx.doi.org/10.4067/S0718$\underline{50062016000600006 .}$.

Woodrow, L. 2017. Motivation in language learning. In Essential competencies for English-medium university teaching (pp. 235-248). Springer, Cham.

https://doi.org/10.1007/978-3-319-40956-6_16 